\title{
Groupware Environments as Action Constitutive Resources: A Social Action Framework for Analyzing Groupware Technologies
}

\author{
OJELANKI K. NGWENYAMA \\ University of Michigan Business School, Ann Arbor, MI, U.S.A. \\ E-mail: Ojelanki_Ngwenyama@ccmail.bus.umich.edu \\ KALLE J. LYYTINEN \\ Computer Science and Information Systems, University of Jyväskylä, SF-40 100 Finland \\ E-mail: Kalle@cs.jyu.fi
}

(Received 14 March 1995; in final form 18 July 1996)

\begin{abstract}
Computer Supported Cooperative Work (CSCW) is a relatively new and dynamic field dealing with the development and use of groupware technologies in organizations. Several frameworks and models have been proposed for studying CSCW, each conveying a different perspective and theoretical basis. Although these frameworks have contributed much to our understanding of the field, they can be criticized for a lack of holistic understanding of the complex social activity that is constitutive of groupwork. This often leads to the failure of otherwise well designed CSCW applications. In this paper we take up this challenge and propose a social action framework for analyzing groupware technologies. The framework is based on Habermas's theory of social action and four action categories, and the idea that groupware applications serve as sets of rules and resources which mediate group interactions. We demonstrate the value of the framework by analyzing a wide range of existing groupware technologies for their appropriateness to specific groupwork situations in terms of their espoused or implicit assumptions of groupwork, and the action constitutive resources they provide. Our analysis points out that a host of current groupware applications can be fairly easily classified and examined by the way they are configured to support different types of social action. It also suggests that, when implementing groupware applications, developers should critically evaluate: (a) the need for supporting a rich variety of action types, (b) the possible role of computer support in the specific groupwork situations, and (c) the underlying assumptions of groupwork embedded in the groupware platform. Finally, we will discuss how the framework can inform future research and development in the field.
\end{abstract}

Key words: Computer supported cooperative work, groupware, group support systems, decision support systems, communication systems, collaboration technology, coordination systems, social action theory

\section{Introduction}

Computer Supported Cooperative Work (CSCW) is a relatively new and dynamic field of research dealing with the development and use of groupware technologies in organizations. In general, groupware technologies ${ }^{\star}$ are expected to support

\footnotetext{
* Though all groupware applications are not limited to groups, as some organizational level applications are included (e.g., Lotus Notes) we believe that our analysis applies to them as well.
} 
groupwork activities, such as planning, coordination, decision making and so on. Research on CSCW is currently diverse and heterogeneous, driven by a wide range of concerns such as new groupware technologies, changes in work organization, and international social and economic transformations. Several frameworks and models have been proposed for studying CSCW (Grief, 1988; Johansen, 1988; Suchman, 1989; Winograd, 1988; Ellis et al., 1991; Schmidt and Bannon, 1992) each conveying a different perspective and theoretical basis. And although these frameworks have contributed much to our understanding of CSCW, a number of researchers (Kling, 1991; Lyytinen and Ngwenyama, 1992; Grudin, 1991) argue that our frameworks still display a lack of understanding of the complex social activity that is constitutive of groupwork. Some socially informed frameworks have emerged (Kuutti, 1992; Winograd, 1988; Suchman, 1989) but more still needs to be done on articulating the complexity of groupwork. For example, Perrin (1991) and Kling (1991) argue, that more research is needed in articulating the complexity of social interactions of groupwork in the organizational context. Greenberg (1991) describes six types of negative effects that can occur when the groupware environment does not fit the groupwork situation and its organizational context. Mandviwalla (1994), and Mandviwalla and Olfman (1994) argue that underlying worldviews of groupware environments do not match the everyday activity of groupwork. Grudin $(1991,1994)$ also suggests that the failure to address the social dimension of groupware implementation leads to user rejection of otherwise well designed applications. As groupware technologies continue to roll out at an ever increasing rate, bringing with them implicit assumptions about groupwork, the need for a framework for systematically analyzing groupware technologies and groupwork situations, and, making acquisition and implementation decisions is becoming more pressing.

\subsection{RESEARCH ON GROUPWARE DESIGN}

A stream of CSCW research (Winograd, 1988; Kuutti, 1992; Schmidt and Bannon, 1992; Suchman, 1989) has focused on defining the problem space of groupwork and suggesting strategies for its computer support. But these studies, although attempting to articulate pertinent social aspects of groupwork in order to inform groupware design and use, have addressed narrow areas of the problem space. For example, Winograd (1988) outlined a framework for research and development of communicative aspects of groupwork based on speech act and conversation theory. In doing so he identifies a set of conversation types that should be supported by groupware. His analysis of conversations is insightful and offers one promising strategy for analyzing some communicative aspects of groupwork and for configuring groupware support for them. What is missing in his framework and analysis is the broader organizational context in which these conversations take place, and how these conversations are intertwined in other types of social activity. Though Winograd emphasizes a set of conversation protocols he does not articulate their 
socially constraining aspects and political implications within the organizational context (Suchman, 1994). Kuutti (1991) and Kuutti and Arvonen (1992) propose a general conceptual scheme for designing groupware based on the idea of the "activity". In their framework an activity is a collective phenomenon which consists of an object and a subject that prevails in a material environment. An activity is realized through the conscious and purposeful use of a tool by a knowledgeable actor (subject) to transform an object-of-work. The fundamental limitation of this framework is that it reduces groupwork to instrumental activity and largely ignores symbolic interaction. Concomitantly, Winograd (1988), Kuutti (1991), and Kuutti and Arvonen (1992) limit the range of social activity either to conversations or instrumental work activities, thus ignoring the social influence process and organizational context. More recently Olson et al. $(1992,1993,1994)$ attacked the problem from a different perspective; they conducted a set of empirical studies of design meetings to try to determine the conceptual structure of the groupwork activity. Their findings suggest that groupwork can be characterized by its activity structure and as such have given impetus to this work. Lastly, Mandviwalla and Olfman (1994) synthesizes the functional features of specific types of groupwork and groupware environments and suggest a set of seven prescriptions for groupware design. While their prescriptions are insightful, they are limited by the underlying assumptions of the groupwork and groupware they analyzed.

A second stream of CSCW research has dealt with the problem of defining the field and suggesting basic questions and puzzles. For example, Schmidt and Bannon (1992) proposed a broad definition of CSCW which acknowledges any endeavor to understand the nature and characteristics of groupwork, and the design groupware technologies as part of the CSCW research agenda. In a similar vein Suchman (1989) suggests that the basic question for CSCW research is how to design computer-based technologies with explicit concern for the socially organized work practices of their intended users. Grudin (1994), also outlines eight puzzles that developers need to address in the design of socially sensitive groupware. Although these definitions, basic questions, and puzzles function as clever and successful devices for building a research community (Latour, 1987), they do not offer much advice on the difficult problems of defining the groupwork problem space and articulating requirements for its support by groupware applications.

Our project here is not to reopen the discussion on what is and what is not CSCW, but to take up the challenge of outlining a framework for analyzing groupwork and groupware environments. The framework we are proposing integrates relevant aspects of groupwork activity suggested by Winograd (1988), Kuutti (1991), Kuutti and Arvonen (1992) and Suchman (1989). It extends the domain of CSCW beyond communicative and instrumental action, but at the same time also deals with the organizational context of social interactions in groupwork. Therefore we call it a social action framework. In this paper our goal is to develop the notions that establish and underlie the framework (Section 2). In addition we demonstrate the value of the framework in analyzing a wide range of groupware technologies 
through uncovering their implicit design assumptions, and the nature and types of resources and rules entailed by these assumptions (Section 3 ).

\section{Groupwork as social action}

The starting point of our discussion is a working definition of groupwork. We define groupwork as: a web of coordinated social actions, ${ }^{\star}$ performed by the participants to achieve a joint outcome. The social actions of groupwork are situated within and normatively regulated by the organizational context. Social action does not ensue without any structural constraints. It presupposes a common medium of communication, culture, shared values, norms, social relations, and a shared understanding of the organizational context; otherwise referred to as structures of legitimation and interpretive schemes (Giddens, 1984). Our definition is deliberately inclusive of a wide range of social encounters, such as, cooperation, coercion, conflict, competition, control, and combat, which can occur in groupwork (Kling, 1991). ${ }^{\star \star} \mathrm{We}$ find that many types of groupwork are essentially "mixed-motive" tasks in which participants try to advance opposing positions and objectives (McGrath, 1984). Groupwork is shaped by: (1) the organizational context, norms and values that regulate the interactions, role responsibilities and expectations of the participants; (2) the participants' needs to negotiate role responsibilities, group norms, and interaction protocols; (3) institutions for sanctioning and legitimizing group behaviors; and (4) organizational motivation, reward and incentive schemes. Whatever the social dynamics of the groupwork, the participants must work together if they wish to achieve an outcome. One common example of groupwork is software development in which different specialists often follow different goals and occasionally find themselves in conflict, but still work together to produce the expected product. Some other examples of groupwork are: business strategy formulation, diplomatic negotiations, curriculum planning, and various types of committee and task force projects.

\subsection{THE SOCIAL ACTION FRAMEWORK}

The framework which we are proposing is informed by Habermas's theory of social action known often as the theory of communicative action (Habermas, 1984, 1987). This theory outlines a set of social action categories and the rules and resources needed to support them in everyday activity. We use these social action categories to extend the conceptual reach of current frameworks, by filling-in missing dimensions of social action and their constitutive resources. In this regard we take a pragmatic approach in adopting Habermas's social action categories as ideal types for theorizing about groupwork and groupware technologies. The rationale

\footnotetext{
* All work done in organizations, including individual work can be characterized in terms of social action, because it is subject to the institutional structures and not soley to the whims of the individual.

$\star \star$ Olson et al. $(1993,1994)$ have also shown all types of social activity can be found in groupwork.
} 
for this theoretical perspective is that it offers a set of conceptual categories for the systematic analysis of social actions involved in groupwork situations and their organizational context. It also provides concepts for critically analyzing the implicit assumptions of groupwork embedded in groupware technologies, and their potential for supporting different social action types. Our study is among a growing body of work that has appropriated Habermas's theory of social action for the study of organizational behavior, and the implementation and use of information technologies in organizations. Forester $(1981,1984)$ used this theoretical perspective to study public sector planning and organizational policies. Mumby (1988) utilized it in an extensive theoretical study of organizational communication, ideology, and culture. Dietz and Widdershoven (1991) also adopted this social action perspective in analyzing communications within electronic media and suggested strategies for overcoming limitations of Coordinator ${ }^{\circledR}$. Ngwenyama and Lee (forthcoming) used the perspective in an empirical study of information richness and social influence in electronic mail communications. More recently Ngwenyama (1996) utilized the same perspective in an empirical study of 17 groupwork situations to define their social action characteristics (see also, Hirschheim and Klein, 1994; Lyytinen, 1986, 1992; Ngwenyama 1987; and Truex, 1994, for other applications). Our use of Habermas's theory of social action differs from these earlier studies in that we apply his concepts as design categories to analyze groupware environments to reveal their embedded assumptions about groupwork.

\subsection{CATEgORIES OF SOCIAL ACTION}

The social action framework distinguishes between four categories of social action, and their basic characteristics, namely, orientation, and constitutive resources (see Figure 1). Two other general features of social action are normative regulation and dramaturgy. The first has to with the tendency of people to conform to social and organizational norms. All social action occurs within an organizational context and is mediated by organizational policies and protocols. The organizational context also provides the actors with a background meaning context for their actions. The latter, dramaturgy, ${ }^{\star}$ has to do with the presentation of self in everyday life. Actors in an organizational context develop strategies for articulating their actions that lend them a personal identity. Action orientation refers to the focus or basic goal of the action type, that which it is trying to achieve. Action constitutive resources refers to the basic resources that an actor needs to skillfully perform the action within the organizational context. Resources include knowledge of the rules and norms of the organizations, social and power relationships, technical knowledge, tools and so on. The skillful execution of each action type assumes a specific set

\footnotetext{
$\star$ Dramaturgy involves performances in which individuals project certain self-images for an audience. Some well known occasions of dramaturgy action are press conferences, photo-opportunities, and television interviews. And although these issues are relevant to the analysis of groupware support a discussion of them would take us too far afield; the reader may consult Goffman (1959) for an extensive discussion.
} 


\begin{tabular}{|c|c|c|}
\hline Category of action & Action orientation & $\begin{array}{l}\text { Action constitutive } \\
\text { resources }\end{array}$ \\
\hline Instrumental & $\begin{array}{l}\text { Transformation, Manipulation, } \\
\text { and Control of Objects }\end{array}$ & $\begin{array}{l}\text { Technical knowledge, } \\
\text { Tools }\end{array}$ \\
\hline Communicative & $\begin{array}{l}\text { Maintaining Understanding } \\
\text { and Coordinating Action }\end{array}$ & $\begin{array}{l}\text { Shared media for } \\
\text { communication, } \\
\text { Knowledge of language, } \\
\text { shared norms, and the action } \\
\text { situation }\end{array}$ \\
\hline Discursive & $\begin{array}{l}\text { Restoring Agreement and } \\
\text { Conditions for Coordinated } \\
\text { Action }\end{array}$ & $\begin{array}{l}\text { Knowledge of rules of } \\
\text { discourse } \\
\& \text { critical debate, } \\
\text { Evaluation protocols, } \\
\text { Shared media for } \\
\text { communication }\end{array}$ \\
\hline Strategic & $\begin{array}{l}\text { Influencing and Transforming } \\
\text { the Behavior of Others }\end{array}$ & $\begin{array}{l}\text { Knowledge of the rules of } \\
\text { process, and the opponent } \\
\text { Items of exchange value, } \\
\text { Shared media for } \\
\text { communication }\end{array}$ \\
\hline
\end{tabular}

Figure 1. Categories of social action and their basic characteristics.

of resources and an understanding of the "rules of the game". Rules of the game refer to explicit and implicit norms governing social interactions and procedures for enacting of specific organizational practices.

\section{Instrumental action}

Instrumental action is goal oriented focusing on the control, manipulation, and transformation of physical artifacts. The enactment of this type of action is dependent upon technical knowledge and tools that together form what Giddens (1984) calls allocative resources (resources that are involved in the generation of power, derived from human dominion over nature). The validity of instrumental action can be judged with regard to its efficiency and effectiveness in achieving the desired end. For the execution of instrumental action, the agent depends upon technical knowledge of cause-effect relationships and skills in the operation of tools. Although the focus of instrumental action is usually inanimate objects, it can be directed at other humans, as is the case of "Theory X" management practices which view workers as objects or immutable constraints to be manipulated in the interest of efficiency (Taylor, 1947). No good examples of CSCW systems that solely implement this orientation could be found in the literature. But the underlying assumptions of highly structured enviroments such as Coodinator make this possible in action situations. And although the ideal of CSCW negates instrumental manipulation of 
participants, this orientation is pervasive in many group processes (Kling, 1991). In groupwork, instances of instrumental action can be observed in joint designs that involve manipulation and transformation of artifacts that are constituted as work-objects. Typical instances are document preparation, software design, and other work activities.

\section{Communicative action}

Communicative action is concerned with achieving and maintaining mutual understanding among participants engaged in coordinated action. It is the "glue" which helps to maintain commitments and the "oil" that keeps group interactions running smoothly. Communicative action is enacted via language and other forms of symbolic interaction and it draws upon interpretive schemes that are mobilized in group interactions. In routine interactions, communicative action implies a claim to validity based on four criteria: comprehensibility, clarity, veracity and sincerity. Communicative activity presupposes a common language, media, and a shared understanding of the organizational context. Communications do not negate the possibility of conflict among the actors. When misunderstandings emerge on what has been communicated, or conflicts about its veracity, appropriateness, and sincerity arise, participants may shift either to discursive or strategic action to restore the understanding through reasoned debate, or to influence and coerce others. Successful communicative action depends heavily on the actors' knowledge of language and the organizational context. By knowledge of language we mean competence in the usage of rules of syntax and semantic and universal rules of pragmatic behavior (Grice, 1975). Communicative action is supported by such technologies as e-mail, bulletin boards or computer conferencing.

\section{Discursive action}

Discursive action is oriented towards developing or restoring the background conditions for collaborative action. When questions are raised about the actions of a participant in a group process, the mode of interaction may shift to discursive action. In such situations, the aim is either to re-establish confidence in what is being said or done, or to find rational explanations for the behavior of the individual. This requires that the participants suspend their immediate objectives in order to search for good reasons to justify or refute the claims which are in question. Discursive activity unfolds through critical debate and argumentation which forms the basis for joint decision making and agreement. It involves the specification and evaluation of goals, objectives, and action-plans, and the achieving a rational consensus on values and norms that will guide collaborative action. Common examples of would-be discursive activity are business strategy formulation, investment planning and public policy planning. Participants of discursive action draw upon a common medium of communication, protocols for interaction, and intuitive (a priori) knowledge of the ground rules of discourse. However, the effectiveness of discursive activity depends heavily upon: (1) well defined principles for evalu- 
ating evidence, defining goals and plans of action; and (2) tools for analysis and evaluation of alternatives. Habermas defines a set of ground rules for guiding ideal type discourse. These are: (1) all participants have an equal chance to make their arguments; (2) only valid reasons are accepted as a basis for an agreement; (3) lying is prohibited; (4) jargon cannot be used unless it is understood by all parties: (5) all commitments made by participants must be honored. Although these rules can never be honored completely in real-world situations due to time, power, and resource constraints, they can be used as a standard to assess or plan discursive activity (cf. Ulrich, 1983). Discursive action is supported by several systems such as meeting support systems and issue based information systems.

\section{Strategic action}

Strategic action is oriented toward achieving advantage over another individual or group. Its focus is on influencing and transforming the behavior of others to conform to the agents' desires or goals. Strategic action is a fundamental aspect of the organizational influence process, often referred as "office politics". It may further be open or covert, depending upon whether the conflict situation is openly admitted or hidden. Social and material resources are constitutive of strategic action in that they are involved in the generation of power and dominion of some actors by others. Such resources include: charisma, social status, authority, and items of exchange value (time, expertise, etc.). The actor involved in strategic action draws upon his/her knowledge of what is feasible to achieve, and of opponents goals, positions, and potential for counter action. Hence, this type of action shares the same basic orientation as instrumental action in deriving its essence from the capabilities of transforming objects. However, it differs in two fundamental ways: (1) Its primary focus is on other humans and in influencing their activities. (2) The agent who engages in strategic action recognizes that the opponent may engage in intelligent counter-action. This Giddens (1994) calls the dialectic of control, because it recognizes that all forms of dependence offer some resources whereby subordinates can influence their superiors. Agents engaged in strategic actions can at the same time draw upon and transform organizational processes, resources, and "the rules of the game" to their advantage (Kipnis, 1976; Salancik and Pfeffer, 1977). Strategic action is deemed legitimate when it conforms to organizational norms, policies, authority structure, and "the unwritten rules of the game". When it does not conform it is considered "dirty tricks". Typical examples of overt strategic activity are negotiation and bargaining. The idea of strategic confrontation is followed in many negotiation support and bargaining systems.

\subsection{THE WEB OF SOCIAL ACTION}

We can now clarify our conjecture that groupwork forms a web of social action. This is entailed by the observation that any moderately complex groupwork situation relies on the execution of all types of social action. In other words, it depends 
simultaneously on structures of signification, domination and legitimation (Giddens, 1984). A specific action type will take the foreground depending on the type of group process involved and its institutional properties. Different institutional properties of group activities needed in software construction illustrate this well. During the project planning process, the focal point of group activity is communicative and strategic: participants discuss and outline project objectives and plan for change contingencies. During design the focus shifts to discursive and communicative action as group members seek to articulate and justify their design proposals and to raise questions about their validity and appropriateness. The design involves a dialogue on design issues, options, and implementation strategy. During the actual software construction, coding and testing, instrumental action plays a dominant role while other types play supporting roles.

\section{Groupware as action constitutive resources}

An important question raised by our analysis is: how can groupware support varieties of social action in a social web? To provide an answer to this question we shall regard groupware applications as action constitutive resources and rules that mediate groupwork (Lyytinen and Ngwenyama, 1992). The configuration of rules and resources is affected by the design of the groupware environment and the organizational context that regulates groupwork. Moreover, this configuration is not static as the groupware evolves while users appropriate and innovate on aspects of the groupware application (Ngwenyama, 1996b). Ultimately, the configuration of rules and resources will determine how well the groupware mediates various types of social action. In general, groupware can provide different types of resources: communicative media around which groupwork can be structured or enabled, or tools to operate on work-objects. Groupware thus provide resources on which participants can draw during their interactions to the extent they serve as media and embody tools for intellectual labor. Groupware applications also embed rules that mediate group interactions and work practices. We distinguish two classes of rules: behavioral rules, such as rules of grammar, organizational policies, explicit norms, and protocols which define and regulate meaningful group behaviors; and technical rules, which are systematic procedures and guidelines that embody action constitutive knowledge to operate tools or interact with the media.

Our division of groupware resources into media, and tools is not architectural in the sense that groupware applications provide either one or the other. As a mediating structure groupware entail both. The distinction is analytical and serves to clarify how designers can analyze and search for different configurations of resources. Groupware serve as media for different types of social activity: (1) in communication they play a role in shaping action situations by providing a channel for communicative, discursive, and strategic interactions; (2) in instrumental work they provide shared work areas within which instrumental action can be enacted. As a communication medium, the groupware can be viewed as a part of the organi- 
zational structure which mediates symbolic interaction. The second aspect of media support is the provision of a shared work space, an kind of a social "ether" within which social actions are propagated and "objects-of-work" are operated upon. From the point of view of tools, groupware provides rules and resources for manipulating and transforming objects-of-work. Tools thus embed technical knowledge in the form of software programs for managing, manipulating, transforming and producing objects-of-work. Examples of such objects-of-work are program code, text, pie charts, histograms, spread sheet tables, and design drawings. With regard to rules, groupware applications embedded: (a) protocols for group interaction which define access, communication patterns and behavior, and schemata for interpreting meaning; and (b) policies which determine what can be done to "work objects", and who is authorized to "work" on them.

\subsection{GROUPWARE SUPPORT FOR SOCIAL ACTION}

Each type of social action assumes a different configuration of resources to ensue successfully. We will now examine what configurations different types of groupware will require, and how these may enable some types of social action while at the same time constraining others. ${ }^{\star}$ This investigation is accomplished by examining typical examples of groupware that have been reported in the research literature. The purpose of this analysis is to demonstrate the value of the framework in interpreting and analyzing how groupware applications constitute specific rules and resources that enable and constrain specific actions. By doing this we are able to see possible limitations of different platforms, to predict their likely impact in specific organizational contexts and groupwork situations, and also to understand the delicate interplay between the organizational context and the groupware.

Our analysis is intended to be broad and general covering a wide range of groupware environments. Moreover, we shall focus on the idealized use situations, that is, so called espoused or intended uses that are reported in the research literature, and the type of social action they place in the foreground. ${ }^{\star \star}$ However, when users appropriate these technologies (DeSanctis and Poole, 1994) actual uses can be much wider than intended uses; and the range of instantiated social actions can be wider than that which the groupware was designed to support. The focus of our analysis is thus on identifying: (a) what rules and resources are "hard/soft wired", in the applications; (b) how they can be adapted to different use situations, and (c) how these configurations can enable and constrain specific group activity. We seek also to identify those resources and rules which lie outside the application, but form part of the background of the group activity. This analysis tries to shed

\footnotetext{
* We do not mean to imply that these groupware environments do not support other types of social action. Rather, we are focusing on the type of action which forms the core of the activity as implied by their implicit assumptions about the groupwork situation that they are intended to support.

$\star \star$ The primary focus of a groupware platform on a specific action type does not negate secondary support for other types of action. For as we have argued earlier, there are critical interdependencies among the action types.
} 


\begin{tabular}{|c|c|c|c|}
\hline $\begin{array}{l}\text { Category of } \\
\text { support }\end{array}$ & $\begin{array}{l}\text { Action } \\
\text { orientation }\end{array}$ & $\begin{array}{l}\text { Constitutive } \\
\text { resources }\end{array}$ & $\begin{array}{l}\text { Groupware } \\
\text { environments }\end{array}$ \\
\hline $\begin{array}{l}\text { Instrumental } \\
\text { groupware }\end{array}$ & $\begin{array}{l}\text { Transformation, } \\
\text { Manipulation, } \\
\text { and Control of } \\
\text { Subjects }\end{array}$ & $\begin{array}{l}\text { Tools, Shared } \\
\text { Material } \\
\text { Technical rules, } \\
\text { Interaction } \\
\text { Protocols }\end{array}$ & $\begin{array}{l}\text { iCASE } \\
\text { Group Editors } \\
\text { Co-authoring } \\
\text { Systems }\end{array}$ \\
\hline $\begin{array}{l}\text { Communicative } \\
\text { groupware }\end{array}$ & $\begin{array}{l}\text { Maintaining, } \\
\text { Understanding } \\
\text { and Coordinating } \\
\text { Action }\end{array}$ & $\begin{array}{l}\text { Communication } \\
\text { Media, Interaction } \\
\text { Interaction } \\
\text { Protocols }\end{array}$ & $\begin{array}{l}\text { CMC, } \\
\text { E-mail } \\
\text { E-conferencing }\end{array}$ \\
\hline $\begin{array}{l}\text { Discursive } \\
\text { groupware }\end{array}$ & $\begin{array}{l}\text { Restoring } \\
\text { Agreement and } \\
\text { Conditions for } \\
\text { Coordinated } \\
\text { Action }\end{array}$ & $\begin{array}{l}\text { Communication, } \\
\text { Media, } \\
\text { DSS Tools } \\
\text { Interaction } \\
\text { Protocols }\end{array}$ & $\begin{array}{l}\text { MSS, } \\
\text { IBIS }\end{array}$ \\
\hline $\begin{array}{l}\text { Strategic } \\
\text { groupware }\end{array}$ & $\begin{array}{l}\text { Influencing } \\
\text { and Transforming } \\
\text { the Behaviour } \\
\text { of Others }\end{array}$ & $\begin{array}{l}\text { Tools, Shared } \\
\text { Material, } \\
\text { Interaction } \\
\text { Protocols, } \\
\text { Communication } \\
\text { Media }\end{array}$ & NSS \\
\hline
\end{tabular}

Figure 2. Examples of groupware and primary action types they target.

light on the distinct qualities that need to be recognized when structuring alternative configurations of resources and rules for different types of groupware. We will also show how our framework can be used to detect inconsistencies in the social design of groupware that embody hidden assumptions about the social activity which they seek to support.

The analysis is organized around four categories characterized by the primary type of action that forms the espoused use of the groupware. These are: (1) Instrumental groupware that support the creation, manipulation, and transformation of objects-of-work, and provide associated tools. Some examples are shared editors, co-authoring systems and iCASE environments. (2) Communicative groupware that support group interactions on the symbolic level, so that group members can understand each other. Some common examples are electronic-mail, electronic conferencing, and bulletin boards. (3) Discursive groupware that support problem solving, argumentation and critical debate. Some examples are group decision support systems, meeting support systems, and issue based information systems. (4) Strategic groupware that support negotiating and bargaining processes. Examples in this category are negotiation support systems (NSS). In the following, 
we briefly overview each class of groupware, and use an example to discuss key characteristics.

\subsubsection{Category 1: Instrumental groupware}

A number of groupware platforms such as group editors, co-authoring systems, and iCASE environments assume that instrumental action forms the focal point of groupwork. In general, these platforms provide tools, media and associated workobjects for group activity which is often characterized as knowledge work. By knowledge work we mean things like joint production of intellectual products such as reports, articles, plans, engineering designs, or software code. The focus here is the efficient manipulation, control and production of such work objects. Perhaps the most common groupware platforms in this category are group editors and authoring systems. Although participants of group writing projects engage the entire spectrum of social action in the course of their work, group writing environments are mostly narrowly focused on supporting instrumental action. These systems provide various tools such as spell-checkers, grammar checkers and document formatters, material and media (i.e. public work space) which can jointly be used for creating and editing documents. Members of the work group can synchronously access documents, reuse and change them, and observe changes being made by others.

Group writing requires a high level of coordination, task planning, information sharing and norm compliance during many cycles of document review. Most co-authoring systems therefore offer some level of support for coordination and norm compliance. But due to the difficulty in formalizing, enforcing and updating such norms in a flexible manner, much of this is left outside to be dealt with in the organizational context. At the software level, solutions to these problems cover different types of rule-based mechanisms that support document protection, update synchronization, conflict resolution, and view convergence. While automatic periodic saving of work-areas during document creation and updating is common in many systems, the most basic form of document protection is version control. Some systems limit update access to a document to one individual at a time, and utilize various coordination mechanisms and priority schemes to resolve access contention. Other systems protect the body of the document, but allow participants to make margin comments or electronic "post-it" notes while the main author is writing it. PrepEditor (Neuwrith et al., 1990) provides a margin work space in which readers can write comments while the "current author" is working on the main text, and a collator for organizing the margin comments and referencing them to the appropriate text location. Others allow multiple entry points to the document, but protect individual cursor locations. This allows a participant to follow behind the main author, editing and cleaning up prose, or simply adding detail to the main text.

Studies have also pointed out that group writing depends not only on the technical support for the writing and editing, but also on ongoing conversations about 
objects-of-work (Ellis et al., 1988). During such interactions work-objects are socially categorized, constructed, identified and their dependencies coordinated to make instrumental transformations possible. This suggests that seamless support for shifting between action types is important to the design of such groupware applications. Therefore, media and rules for symbolic interaction that enable the instrumental action ought to be provided in the groupware environment. Task planning, role responsibility, information sharing and document review are activities generally neglected in this class groupware. Although few systems, like QUILT (Leland et al., 1986) allow the users to define role responsibilities, modify readwrite access to the shared material, and, to create distribution lists for information sharing. They also provide tools to assist task planning. Some systems such as Capture Lab (Mantei, 1988) manage access to shared work spaces via electronic requests mechanisms which implement a communicative action sub-component to coordinate activities. When a participant wishes to gain access to the shared work space he/she electronically notifies the current author, and waits for access to be relinquished.

Overall, groupware for instrumental action offer little support for communicative, discursive, and strategic action. These action types are handled outside the application by organizational arrangements and co-location of group members. Co-location provides participants possibilities discuss and debate objects-of-work (Conklin and Begeman, 1988). Two types of information technologies, desktop video windows and hypertext, could extend these support capabilities to cover other action types. Desktop video could extend the bandwidth for social action in geographically distributed writing projects thus, eliminating need for co-location. Moreover, hypertext could extend document annotation and support for the maintenance of the history of discursive activity thus, recording ideas behind the final document.

\subsubsection{Category 2: Communicative groupware}

A wide range of computer mediated communication (CMC) groupware assume communicative action as the focal point of groupwork. These groupware platforms place in the foreground a medium for communication thus hiding in the background tools for preparing, distributing and managing messages as objects-of-work. CMCs can be divided into three classes based on the nature of communications they support: (1) electronic mail; (2) electronic conferencing; and (3) electronic news. Each class of CMCs assembles different configurations of rules and resources to support these communication activities. Electronic mail systems provide a communication medium to help participants create and transmit relatively unstructured messages, and tools to organize and manage conversations among individuals and loosely organized groups. Electronic conference systems provide a public arena (shared media) for exchanging information and opinions, and tools for establishing and managing continuing conversations, among specific group participants. Electronic 
news systems provide a public space for individuals to broadcast news, and share information. All these CMC environments are capable of embedding organizational rules and norms. The level of embedding of organizational rules depends on the CMC system, its implementation, and the management philosophy of the organization. Likewise, the level of control that users can exercise when configuring their CMC support for the group situation depends on the particular system, how it is "organizationally" implemented, and the management philosophy.

Many systems, such as Lotus Notes ${ }^{\circledR}$ (1989) and TEIES (Turoff 1989) offer a wide range of tools to configure group specific applications. Using such systems, organizations can micro-manage electronic communications down to the most basic level of message formats and distribution patterns. More sophisticated systems, such as CHAOS (DeCindio et al., 1986) COMTRACK (Koo, 1988), Coordinator $^{\circledR}$ (Flores et al., 1988) and OVAL (Malone et al., 1992) seek to support organizational coordination and management by offering means to inscribe stringent control and management of conversations and speakers' intentions. This is achieved by providing software tools that record and monitor commitments, define and broadcast role responsibilities and expectations, and coordinate contingent activities. For example, the well known conversation management tool Coordinator ${ }^{\circledR}$ (Flores et al., 1988) supports coordination and tracking of requests, commitments, and their fulfillment. It also imposes a communication pattern based on the structure of the "conversations for action". In CHAOS, users can also delegate responsibilities, revoke responsibilities, create fields of responsibility, and so on.

Even though communicative action is the central focus of the CMC applications, other social action types that play supporting roles in communications are generally supported. For example, when communication breaks down due to misunderstanding, or when the veracity of participant's statement is challenged the mode of group interaction can switch to discursive and/or strategic action. Several CMCs provide support for these types of social action. For example, ConversationBuilder (Carroll, 1992) and Strudel (Shepherd et al., 1990) provide hypertext support for tracking arguments and clarifying assumptions. Although CMCs offer no specific support for strategic action, users have been able to appropriate these systems for bargaining (Turoff, 1985), and interpersonal influence processes (Phillips, 1989). One limitation of current CMC environments is their "media richness". For example, in face-to-face communication, individuals can use gestures and body language to communicate their meaning (dramaturgy). Since most CMCs are limited to written media and users are often not co-located this constrains the "semantic richness" of symbolic interaction. Individuals have to be very innovative to express their personal styles. Experiments with multi-media CMC environments such as desktop video windows have demonstrated, however, that richer media can significantly improve group interaction (Ragan and Vin, 1991; Ishii and Kabayashi, 1992).

Current CMCs have been found inadequate for several group tasks due to their limited focus on communicative functions and very strict, closed "soft-wired" rule 
set. For example, reported failures of Coordinator (Durham, 1988, Grantham and Carasik, 1988) have been attributed to the fact that it embodies a closed set of linguistic rules that cannot be negotiated during its use. However, negotiation is necessary for shifting from communicative action to discursive and strategic action when communications break-down. Yet, this feature of communication is seldom supported in CMCs (Robinson, 1993; Tatar et al., 1992). In most cases, the success of Coordinator has not been due to its explicit support of the pragmatic rules of the conversation, but to the flexible tools (qua resources) it provides to manage and store messages and to send them over a number of different computer platforms (DeMichelis, 1993; Krcmar, 1993).

\subsubsection{Category 3: Discursive groupware}

In discursive groupware the focal point of groupwork support is discursive action. Accordingly, this class of groupware provide means and arenas for open and critical problem exploration and decision making. ${ }^{\star}$ Discursive groupware environments can be classified into three types based on their functionality and support focus: (1) Group Decision Support Systems (GDSS), (2) Meeting Support Systems (MSS), and (3) Issue Based Information Systems (IBIS). GDSS and MSS groupware tend to assume traditional hierarchical models of groupwork, such as the management meeting. These are fairly well defined in practice. By "meetings" we mean time limited, goal oriented, face-to-face encounters of two or more participants which have specified agendas and role structures. These systems can be defined as discursive because they seek to relax some of the limitations and constraints posed by the hierarchical management meeting such as: (a) seriality and limitations to air time, (b) control over topics and viewpoints followed, and (c) the time spent on rational debate and analysis. The systems provide a public work space and tools for anonymous problem representation, problem structuring and analysis. They also provide private individual work spaces for preparing positions, analyzing data, and note taking. GDSSs support participants in developing shared representations of problems and solutions, and evaluating decision alternatives. They also provide anonymity and parallel processing which help to amplify "rationality" of the group process and reduce management bias (Dennis et al., 1988). MSSs are designed around dedicated and specially equipped meeting rooms where personal computers are linked together and to a public electronic blackboard. They provide "public electronic domains" in which ideas, arguments, and opinions can be expressed, organized, and developed. MSS's are sometimes considered second generation GDSS, because they subsume much of the functionality of GDSS.

The extent to which organizational rules are embedding in GDSS and MSS environments is relatively high. Users have little flexibility to configure rules and

\footnotetext{
* Attempts have also been made to extend other groupware platforms, such as electronic mail and conferencing systems, with formal group structuring techniques such as the Delphi Method and Nominal Group Technique in order to provide support for discursive action (Turoff and Hiltz, 1982).
} 
resourcess for specific situations. For example, users cannot configure additional social relations, or define access rights to specific information spaces based on the needs of the groupwork situation. Moreover, though GDSS and MSS provide resources for all action types, their fundamental rationale is to improve the rationality of the interactions. Further, they are neither neutral nor open (ideal speech) environments for collaboration. One reason for this is that they are designed around the notion of the structured meeting, with closed agendas and a hierarchical structure epitomized by a chairperson. They also entail well socialized meeting protocols (Roberts Rules etc.). In addition, much of the social structure is embedded in the meeting room architecture and seating arrangements. A second limitation of these environments is that they fix the time and place of group activity and require an elaborate social process for its initiation and execution.

The philosophy of IBIS ${ }^{\star}$ groupware differs from that of GDSS and MSS. The IBIS model (Kunz and Rittel, 1970) views problem solving as an argumentation process. The argumentation process is represented as a complex network of issues where each issue forms a root node from which a sub-network of positions and arguments may evolve. Each problem situation is said to have key issues, and each issue may have several positions, articulated statements, or assertions which resolves an issue. Each position may have one or more arguments in support of, or in objection to, it. IBIS provides a less structured environment for problem exploration and decision making and supports long term debate over complex, ill-structured problems. An example of this type of environment is gIBIS (Conklin and Begemann, 1988), a "design journal" which supports software design meetings. Conceptually, gIBIS views the software design process as a multi-participant collaborative discourse. It provides support for: (1) the capture of design history and rationale, that is, decisions, trade-off analysis, and rejected design options which provide justifications for design decisions; and (2) a computer mediated environment to support design conversations. The gIBIS serves as a storehouse for design documents, interview reports, design reviews, early notes, and minutes. The level of embedding of behavioral rules in IBIS groupware is generally low, and user access to configure group problem solving processes is more open relative to GDSS and MSS. A typical IBIS dialogue may begin by some member of a work group (e.g., a strategy formulation team) posing a basic question, such as: how should we define our core business? The member may raise issues such as: (1) Should it be that narrow area within which the company was launched? (2) Should it be the area which currently makes the greatest contribution to net income? Most likely, other members of the work group will respond to these issues by stating positions and arguments for and against them. They may raise other issues as well.

\footnotetext{
* More recently, two other IBIS-like groupware environments have been proposed to support discursive activity: CONEX (Hahn et al., 1990) which is based on Toulmin's (1958) theory of argumentation; and SPIDER (Boland et al., 1992) which is based on cognitive mapping. But although these environments share a set of core characteristics, they differ in their discourse support strategies.
} 
Groupware environments for discursive action have mostly been designed with a fairly narrow view of the web of social action. For example, there is little support to resort to strategic or communicative action in situations where such need arises. For example, most MSS and GDSS environments do not offer mechanisms to build up coalitions, recognize political tactics, and so on (Lyytinen et al., 1994). The high level of rule embedding in these groupware can constrain such action. Yet, the dependency of discursive action on strategic and communicative action is obvious. To take another example, during discursive activity there is often a need to come to a shared understanding of the situation, to clarify vocabularies (which often require theasuri and other means of clarification) and to coordinate different tasks and activities.

\subsubsection{Category 4: Strategic groupware}

Few groupware platforms place strategic action in the foreground. Generally, this necessitates the support of negotiation and bargaining processes which are characterized by goal conflict, deception, unequal distribution of information and power. Although such processes are very common in organizational life, there is not much research on this type of groupwork support. One reason for this is the inherent bias of CSCW researchers to imagine groupwork in terms of harmony and collaboration. The second one is the inherently complex and fluid nature of rules and resources that are drawn in such interactions (Lyytinen et al., 1994). Currently, one groupware platform that seeks to supports this class of groupwork activities is Negotiation Support Systems (NSS). Research and development on NSS groupware has been growing slowly since its inception in the early 1980s. The basic objective of this research is to increase the "productivity" of mixed-motive group interaction (McGrath, 1984) by providing rules and resources for improving: (1) joint problem representation and analysis; (2) negotiation process management; (3) conflict management; (4) maintenance and recognition of the relationship between negotiators; (5) the conflict resolution skills of mediators; and (6) the quality and level of acceptance of negotiated agreements.

One of the earliest examples of NSS groupware is the Conflict Analysis Program (Fraser and Hipel, 1981) which helps arbitrators analyze feasible agreements based on participants' preferences, identify and eliminate infeasible proposals, and assess the stability of negotiated agreements. Another example is the Automated Decision Conferencing System (Quinn et al., 1985) which assists negotiators in disaggregating issues, creating alternative proposals, and anticipating the proposals of opponents. Other examples which support negotiators are NEGO (Kersten, 1985) and NEGOPLAN (Matwin et al., 1989). NEGO is designed to help negotiators evaluate compromises and identify "common ground," while NEGOPLAN helps them prioritize their goals and assess the implications of different outcomes. In some recent studies (Lyytinen et al., 1993, 1994) negotiation support systems have been designed for multilateral diplomatic groups. These studies analyze, in 
more detail, classes of behavioral rules and contextual resources involved in the diplomatic encounters, that are also typical to complex bargaining situations.

Another widely reported NSS groupware application in this category is MEDIATOR (Jarke et al., 1987). It was developed to support a human mediator in complex negotiations. MEDIATOR provides negotiators with a set of analysis tools, private work spaces, and access to data models. It provides the mediator with a public work space for joint problem representation, and tools for view integration, analysis, and process management. By integrating the views of opponents, the mediator can build a joint representation of the problem and analyze it in order to identify "areas of agreement" which could serve as a starting point for consensus building. He or she can also identify coalitions and feasible agreements, and develop strategies for moving the group toward consensus. Recently, Ngwenyama et al. (1996) have developed a set of techniques for analyzing preference data collected in GSS and supporting consensus formation. These techniques assist facilitators in identifying and reporting to the group what are the problematic issues, and the collective and individual positions on all issues.

The difficulty of designing strategic groupware environments is partly due to the complex and derivative nature of these types of group processes. Strategic action leads to very complex and fluid interactions with shifting behavioral rules, because the participants often transform the "rules of the game" during the unfolding of the process. Accordingly, there is a need for very flexible and open-ended designs of such environments where most of the organizational rules and resources are not embedded in the system, but form part of the groupwork situation. The derivative nature implies that the system must also address the need to support communicative, discursive and instrumental actions in the groupwork situation. This can lead to complex and very extensive systems with contradictory operating procedures.

\section{Conclusions}

In this paper we outlined a social action framework and used it to analyze a range of groupware platforms to expose how they embed assumptions about specific action types and orientations. In doing so we have opened up new possibilities for groupware research by providing a set of categories for investigating the "social action" in groupwork and what is required for its computer support. We believe that the set of concepts outlined here offers a fruitful perspective from which new insights on the role of computer support in groupwork situations can be developed. In this paper our basic objective was simply to articulate some important aspects of groupwork from the social action perspective and to demonstrate its value in analyzing groupware technologies. In particular, we examined those action constitutive rules and resources that a wide range of current groupware platforms provide, and discussed some of their limitations in supporting the whole spectrum of groupwork. Thereby demonstrating how the social action framework can help to articulate pertinent issues in groupware research and to focus it accordingly. 
Although we did not set out to exhaustively classify existing groupware, the sample that we examined seem to logically fall into specific social action categories. Furthermore, from the social action perspective we addressed the limitations of the communicative and instrumental frameworks discussed in Section 2 by showing: (a) how instrumental activity is necessarily linked with symbolic group interactions, and (b) how symbolic interactions are too narrowly viewed in instrumentalist conceptions of groupwork. Moreover, our analysis recognizes important limitations in current groupware platforms that are targeted to support the other two types of action. One such limitation, is placing the primary focus on those rules and resources embedded in the groupware and ignoring: (a) how they interact with the rules and resources in the groupwork situation and its organizational context, and (b) how the groupwork situation and organizational context changes over time.

Despite the value of this first level analysis of groupware platforms vis-à-vis the social action framework, a lot more can be gained from it. Here we have three specific tasks in mind: (1) analyzing groupwork situations and deriving requirements for their support; (2) making decisions about groupware technology acquisitions; and (3) analyzing organizational experiences with groupware technology. With regard to the first we do not currently know how groupware applications need to be configured to cover the range of action types and thereby to meet the work challenges faced by organizational members. We need to develop a more detailed and systematic way of characterizing groupwork situations and their rule-resources configurations so that we can derive specific requirements to support these situations. In addition, it is not clear, how changes in the rules and resources embedded the groupware will change the "parameters" of social action (i.e., the type of knowledge necessary to engage successfully in that type of action). But in general, changes in groupware can lead to unintended changes in the use of the groupware. In reference to the second issue: what types of rules and resources are necessary for the successful realization of each action type, there is a dearth of empirical studies to answer this question. This makes the decision to adopt groupware technology that fit complex groupwork situations encompassing several action types a difficult one. So far, the focus of groupware research has been on applications that target individual action types, whereas our analysis suggests that it is the total web of interactions and social relations that affects the uses of groupware applications, and this should also be taken into account in groupware implementations.

Finally, several new topics have entered into our empirical research agenda due to the insights obtained by the framework. We intend to conduct field studies to examine how different classes of groupware mediate different types of action, and how the interactions with the technology that constitute specific rule and resource sets enable and/or constrain users. For example, we shall investigate how deployed iCASE environments inhibit communicative action which in turn inhibits effective coordination of instrumental action. To understand such dynamics better we are also studying design meetings to understand how episodes of group activity are strung together by rapid shifts from one action type to another. However, 
several other types of group processes need to be studied, in order to develop a better understanding of groupwork and behaviors. Such an understanding would illuminate specific aspects of groupwork situations: (a) what types of action can and need to be supported; (b) how and why actors change their orientations and how this should be taken into account in the groupware design; and (c) in what manner different action types can enter the foreground of groupwork. We expect this to have significant implications not only for determining groupware requirements, but also for understanding how users appropriate applications and innovate upon them in situations for which they were not designed. Examples of this are discussed in Phillips' (1989) study on how electronic mail which was supposed to support genuine communications was often used strategically to influence and manipulate individuals. We want to analyze the latent potential for alternative uses which may be hidden in groupware, and how this potential might be appropriated by different types of users. On this latter issue we are studying two groupware environments to determine: (a) their strengths and limitations in supporting a range of social action; and (b) the extent to which their strengths and limitations depend on users ability to modify the applications.

\section{References}

Boland, R. J., R. V. Tenkasi, and D. Te'eni (1994): Designing Information Technology to Support Distributed Cognition. Organization Science, vol. 5, no. 3, pp. 456-475.

Carroll, A. M. (1992): The ConversationBuilder Kernel and Applications. Ph.D. thesis University of Illinois.

Conklin, J. and M. L. Begeman (1988): gIBIS: A Hypertext Tooling for Exploratory Policy Discussion. In Proceedings the ACM Conference on CSCW. New York: ACM Press, pp. 140-152.

DeCindio, F., G. DeMichelis, C. Simone, R. Vasslo, and A. Zanaboni (1986): CHAOS as a Coordination Technology. In Proceedings of CSCW.

DeSanctis, G. and M. S. Poole (1994): Capturing the Complexity in Advanced Technology Use: The Adaptive Structuration Theory. Organization Science, vol. 5, no. 2, pp. 121-147.

Dietz, J. L. G. and G. A. M. Widdershoven (1991): Speech Acts or Communicative Action. In Proceedings of the Second European Conference on CSCW, pp. 235-248.

Durham, T. (1988): Organizational Dinosaurs Take on a Human Face. Computing, vol. 3, no. 11.

Ellis, C. A., S. J. Gibbs, and G. L. Rein (1988): Groupware: The Research and Development Issues. MCC Technical Report no. STP-414-88.

Flores, F., M. Graves, B. Hartfield, and T. Winograd (1988): Computer Systems and the Design of Organizational Interaction. ACM Transactions on Office Information Systems, vol. 6, no. 2, pp. 153-172.

Forester, J. (1983): Critical Theory and Organizational Analysis. In G. Morgan (ed.): Beyond Method. Beverly Hills, CA: Sage.

Forester, J. (1982): Know Your Organizations: Planning and the Reproduction of Social and Political Relations. Plan Canada, vol. 22, pp. 3-13.

Foster, G. and M. Stefik (1986): Cognoter, Theory and Practice of Collaborative Tool. In Proceedings of the CSCW'86, pp. 7-15.

Frazer, N. and K. Hipel (1981): Computer Assistance in Labor Management Negotiation. Interfaces, vol. 11, pp. 22-29.

Goffman, E. (1959): The Presentation of Self in Everyday Life. New York: Anchor Books.

Grantham, C. K. and R. P. Cararsik (1988): Phenomenology of Computer Supported Cooperative Work. Berkeley. CA: Interpersonal Software. 
Greenberg, S. (1991): Personalizable Groupware: Accomodating Individual Roles and Group Differences. In ECSCW'91 Proceedings of the 1991 European Conference on CSCW. Dordrecht: Kluwer Academic Publishers, pp. 17-32.

Grice, H. P. (1975): Logic and Conversation. In P. Cole and J. Morgan (eds.): Syntax and Semantics, Vol. 3, Speech Acts. New York: Academic Press, pp. 41-58.

Greif, I. (ed.) (1988): Computer Supported Cooperative Work: A Book of Readings. San Mateo, California: Morgan Kaufmann Publishers.

Grudin, J. (1994): Groupware and Social Dynamics: Eight Challenges for Developers. Communications of ACM, vol. 37, no. 1, pp. 93-105.

Grudin, J. (1991): CSCW Introduction. Communications of the ACM, vol. 34, no. 12, pp. 31-34.

Habermas, J. (1972): Knowledge and Human Interest. Boston: Beacon Press.

Habermas, J. (1984): The Theory of Communicative Action: Reason and the Rationalization of Society, Vol. I. Boston: Beacon Press.

Habermas, J. (1984): The Theory of Communicative Action: Lifeworld and System, Vol. II. Boston: Beacon Press.

Hirschheim, R. and K. Klein (1994): Realizing Emancipatory Principles in Information Systems Development: The Case for ETHICS. MIS Quarterly, vol. 18, no. 1, pp. 83-109.

Ishii, H. and M. Kobayashi (1992): Clearboard: A Seamless Medium for Shared Drawing and Conversation with Eye Contact. In Proceedings of Conference on Human Computer Interaction.

Jarke, M., M. T. Jelassi, and M. F. Shakuni (1987): MEDIATOR: Towards a Negotiation Support System. European Journal of Operations Research, vol. 31, pp. 314-334.

Johansen, R. (1988): Groupware: Computer support for Business Teams. New York: Free Press.

Kersten, G. (1985): NEGO-Group Decision Support System. Information and Management, vol. 8, pp. 237-246.

Kipnis, D. (1983): The Use of Power. In R. W. Allen and L. W. Porter (eds.): Organization Influence Process. Foresman, IL: Scott, pp. 18-35.

Kling, R. (1991): Cooperation, Coordination and Control in Computer Supported Work. Communications of ACM, vol. 34 , no. 12 , pp. 83-88.

Koo, C. (1988): A Commitment-Based Communication Model for Distributed Office Environments. In Proceedings of CSCW, pp. 291-298.

Kunz, W. and H. Rittel (1980): Issues as Elements of Information Systems. Working Paper no. 131. Institute of Urban \& Regional Development, University of California, Berkeley.

Kuutti, K. (1991): The Concept of Activity as a Basic Unit for CSCW Research. In Proceedings of the 2nd ECSCW, pp. 249-264.

Kuutti, K. and T. Arvonen (1992): Identifying Potential CSCW Applications by Means of Activity Theory Concepts: A Case Example. In Proceedings of Conference on Computer Supported Cooperative Work, pp. 233-240.

Latour, B. (1987): Science in Action: How to Follow Scientist and Engineers Through Society. Open University Press, Milton Keynes.

Leland, M. D. P., R. S. Fish, and R. E. Kraut (1988): Collaborative Document Production Using Quilt. In Proceedings of Conference on Computer Supported Cooperative Work, pp. 206-215.

Lotus (1989): Lotus Notes Users Guide. Cambridge, MA: Lotus Development Corp.

Lyytinen, K. (1986): Information Systems Development as Social Action: Framework and Critical Implications. Ph.D. dissertation, Department of Computer Science, University of Jyvaskyla, Finland.

Lyytinen, K. (1992): Critical Theory and Information Systems. In M. Alvesson and H. Willmott (eds.): Critical Management Studies. Beverly Hills, CA: Sage.

Lyytinen, K. J. and O. K. Ngwenyama (1992): What Does Computer Support for Cooperative Work Mean? A Structurational Analysis of Computer Supported Cooperations Work. Accounting Management and Information Technology, vol. 2, no. 1, pp. 19-38.

Lyytinen K., P. Maaranen, and J. Knuuttila (1993): Unusual Business or Business as Usual: An Investigation of Meeting Support Requirements in Multilateral Diplomacy. Accounting, Management and Information Technologies, vol. 3, no. 2, pp. 97-119. 
Lyytinen K., P. Maaranen, and J. Knuuttila (1994): All Groups Are Not the Same: An Analysis of Group Behaviors in Electronic Meeting Systems. Computer Supported Cooperative Work, vol. 2, no. 3, pp. 261-284.

Mandviwalla, M. (1994): The Worldview of Collaborative Tools. Electronic Journal of Virtual Culture, vol. 2, no. 2. listserv@kentvm.kent.edu

Mandviwalla, M. and L. Olfman (1994): The What Do Groups Need: A Proposed Set of Generic Groupware Requirements. ACM Transactions on Human Computer Interaction, vol. 1, no. 3, pp. 245-268.

Mantei, M. (1988): Capturing the Capture Lab Concepts: A Case Study in the Design of Computer Supported Meeting Environments. In CSCW'88 Proceedings of the Conference on Computer Supported Cooperative Work, pp. 257-270.

Markus, L. M. and T. Connolly (1990): Why CSCW Applications Fail: Problems in the Adoption of Interdependent Work Tools. In CSCW'90 Proceedings of Conference on Computer Supported Cooperative Work. New York: ACM Press, pp. 371-380.

Matwin, S., S. Sxpakwicz, G. Kersten, and W. Michalowski (1989): NEGOPLAN: An Expert System Shell for Negotiation Support. IEEE Expert, vol. 4, pp. 50-62.

McGrath, J. E. (1984): Groups: Interaction and Performance. Englewood Cliffs, NJ: Prentice-Hall.

Mumby, D. K. (1988): Communication and Power: Discourse Ideology and Domination. New Jersey: Ablex.

Neuwirth, C. M., D. S. Kaufer, R. Chandhok, and J. H. Morris (1990): Issues in the Design of Computer Support for Co-Authoring and Commenting. In CSCW'90 Proceedings of the Conference on Computer Supported Cooperative Work. New York: ACM Press, pp. 183-195.

Ngwenyama, O. K. (1987): Fundamental Issues of Knowledge Acquisition: Toward a Human Action Perspective of Knowledge Systems. Ph.D. dissertation, Thomas J. Watson, School of Engineering, SUNY.

Ngwenyama, O. K. (1996a): On the Phenomenology of Group Processes: A Social Action Analysis of Software Design Meetings. Working Papers, University of Michigan Business School.

Ngwenyama, O. K. (1996b): Breakdowns and Innovations in Computer Mediated Work: Groupware and the Reproduction of Organizational Knowledge. Submitted for publication.

Ngwenyama, O. K., N. Bryson, and A. Mobolurin (1996): Supporting Facilitation in Group Support Systems: Techniques for Analyzing Consessus Relevant Data. Decision Support Systems, vol. 16, no. 2, pp. 155-168.

Ngwenyama, O. K. and A. Lee. Information Richness in Electronic Communication: Critical Theory and The Contextuality of Meaning. MIS Quarterly (forthcoming).

Olson, G. M., J. S. Olson, M. Storrosten, M. Carter, J. Herbselb, and H. Rueter (1994): The Structure of Activity during Design Meetings. In T. P. Moran and J. M. Carroll (eds.): Design Rationale: Concepts, Techniques and Use. Hillsdale, NJ: Lawrence Erlbaum Associates, pp. 217-239.

Olson, J. S., G. M. Olson, M. Carter, and M. Storrosten (1992): Small Group Design Meetings: An Analysis of Collaboration. Human Computer Interaction, vol. 7, pp. 347-374.

Olson, J. S., G. M. Olson, M. Storrosten, and M. Carter (1993): Groupwork Close Up: A Comparison of the Group Design Process with and without a Simple Group Editor. ACM Transaction on Information Systems, vol. 11, pp. 321-348.

Orlikowski, W. J. (1992): The Duality of Technology: Rethinking the Concept of Technology in Organizations. Organization Science, vol. 3. no. 3, pp. 398-427.

Quinn, R., J. Rohrbough, and M. McGrath (1985): Automated Decision Conferencing: How It Works. Personnel, vol. 62, pp. 49-55.

Perin, C. (1991): Electronic Social Fields in Bureaucracies. Communications of the ACM, vol. 34, no. 12 , pp. $75-82$.

Phillips, S. R. (1989): Electronic Persuasion: The Uses of Electronic Mail for Interpersonal Influence in Organizations. Ph.D. dissertation, University of California.

Robinson, M. (1993): Designing for Unanticipated Uses. In ECSCW'93 Proceedings of Third European Conference on CSCW, 13-17 September 1993, Milan, Italy. Dordrecht: Kluwer Academic Publishers, pp. 187-202.

Salancik, G. R. and J. Pfeffer (1977): Who Gets Power and How They Hold on to It: A Strategic Contingency Model of Power. Organizational Dynamics, Winter. 
Shepherd, A., N. Myers, and A. Kuchinsky (1990): Strudel: An Extensible Electronic Conversation Toolkit. In CSCW'90 Proceedings of the Conference on CSCW. New York: ACM Press, pp. 93104.

Schmidt K. and L. Bannon (1992): Taking CSCW Seriously: Supporting Articulation Work. Computer Supported Cooperative Work, vol. 1, no. 1, pp. 7-40.

Suchman, L. (1989): Notes on Computer Support for Cooperative Work. WP-11, Department of Computer Science, University of Jyvaskyla, Jyvaskyla, Finland.

Suchman, L. (1994): Do Categories Have Politics? The Language/Action Perspective Reconsidered. Computer Supported Cooperative Work, vol. 2, no. 3, pp. 177-190.

Taylor, F. W. (1947): Scientific Management. New York: Harper.

Turoff, M. (1985): Information and Value: The Internal Information Marketplace. Journal of Technology Forecasting and Social Change, vol. 27, no. 4, pp. 257-373.

Ulrich, W. (1983): Critical Heuristics of Social Planning. Bern: Haupt.

Winograd, T. (1988): A Language-Action Perspective on the Design of Cooperative Work. STANCS-87-1158, Department of Computer Science, Stanford University, Stanford. 